\title{
Time-dependent simulations of electron transport through a quantum ring: Effect of the Lorentz force
}

\author{
B. Szafran ${ }^{1,2}$ and F. M. Peeters ${ }^{1}$ \\ ${ }_{1}^{1}$ Departement Fysica, Universiteit Antwerpen (Campus Drie Eiken), Universiteitsplein 1, B-2610 Antwerpen, Belgium \\ ${ }^{2}$ Faculty of Physics and Applied Computer Science, AGH University of Science and Technology, aleja Mickiewicza 30, \\ 30-059 Kraków, Poland
}

(Received 11 March 2005; revised manuscript received 3 May 2005; published 3 October 2005)

\begin{abstract}
The time-dependent Schrödinger equation for an electron passing through a semiconductor quantum ring of nonzero width is solved in the presence of a perpendicular homogeneous magnetic field. We study the effects of the Lorentz force on the Aharonov-Bohm oscillations. Within the range of incident momentum for which the ring is transparent at zero magnetic field, the Lorentz force leads to a decrease of the oscillation amplitude, due to the asymmetry in the electron injection in the two arms of the ring. For structures in which the fast electrons are predominantly backscattered, the Lorentz force assists in the transport, producing an initial increase of the corresponding oscillation amplitude. Furthermore, we discuss the effect of elastic scattering on a potential cavity within one of the arms of the ring. For the cavity tuned to shift maximally the phase of the maximum of the wave packet we observe a $\pi$ shift of the Aharonov-Bohm oscillations. For other cavity depths oscillations with a period of half of the flux quantum are observed.
\end{abstract}

DOI: 10.1103/PhysRevB.72.165301

PACS number(s): 73.63.Kv

\section{INTRODUCTION}

The wave function of an electron passing along a path $l$ acquires a phase shift ${ }^{1}$ from the vector potential $\mathbf{A}$ given by $\phi=\left(2 \pi / \Phi_{0}\right) \int_{l} \mathbf{A} \cdot d \mathbf{x}\left(\Phi_{0}=h / e\right.$ is the flux quantum). In a ring configuration the Aharonov-Bohm (AB) effect produces a measurable interference ${ }^{1}$ due to the relative phase shifts of the wave function going through the arms $\Delta \phi=2 \pi \Phi / \Phi_{0}$, where $\Phi$ is the magnetic field flux through the area inside the ring. Oscillations of the electric properties with period $\Phi_{0}$ were detected in metal ${ }^{2}$ and semiconductor rings. ${ }^{3-7}$

The theory ${ }^{8-10}$ of the $\mathrm{AB}$ conductance oscillations was developed in a strictly one-dimensional model in which the magnetic field is inaccessible for electrons and the only effect of the vector potential is the $A B$ phase shift ${ }^{8-11}$ In fact the experiments ${ }^{2-7}$ are performed in homogeneous magnetic fields and the leads have a finite width so the magnetic field declines the paths of the current flow. Nevertheless, the scattering matrix theories ${ }^{8,9}$ assumed explicitly transport symmetry with respect to the arms of the ring. Comparable amplitudes for wave function in both arms of the ring were also assumed in the derivation ${ }^{10}$ of the multichannel AB conductance formula. The theories of Refs. 8-10 addressed metals for which, as we discuss below, these assumptions are justified, since the Lorentz force has a negligible effect on trajectories of heavy-effective-mass electrons traveling with high Fermi velocities. This is no longer true for semiconductor structures.

The purpose of the present paper is to describe the effect of the Lorentz-force-related deformation of the electron trajectories on the $\mathrm{AB}$ effect in a semiconductor quantum ring. The effect of the Lorentz force was discussed for biprism diffraction experiments in vacuum. ${ }^{12}$ The envelope of the interference pattern for an electron traveling in the magnetic field is shifted by the magnetic force according to the classical laws ${ }^{12}$ following the Ehrenfest theorem. The electron trajectories declined by the magnetic field were also studied for the injection through a semiconductor quantum point contact. ${ }^{13}$ The effect of the magnetic field on the electron trajectories in quantum rings was previously addressed in Ref. 14. However, the boundary conditions applied in this paper $^{14}$ are not best suited for the discussion of the Lorentz force effect since the wave function for the outgoing wave is not associated with the current flowing out of the ring (see Sec. IV) and the "incident" electron is not necessarily moving toward the ring. This is due to the current flowing in the opposite directions at the edges of the leads in the eigenstates at high magnetic fields. The problem of the changing orientation of the electron velocity across the leads is in the present paper neutralized by the time-dependent approach. In this approach the incoming lead is clearly defined by the applied initial condition for the localization of the wave packet. The present calculations are performed in a basis of Gaussian functions with embedded gauge invariance. We consider the lowest subband transport and neglect inelastic scattering effects. We demonstrate that the Lorentz force produces a preferential injection of the electron wave packet in one of the arms of the ring. The injection imbalance grows monotonically with the external magnetic field and eventually leads to a suppression of the AB oscillations at high magnetic fields. We find that for high incident momenta the Lorentz force can be necessary to guide the transport of electrons, which are otherwise, in the absence of the magnetic field, backscattered to the incoming lead. When the transport window is opened for the fast electrons, they are directed to both the arms of the ring and the corresponding AB oscillations amplitude initially increases with the magnetic field.

The real rings are never ideally clean, and the transport is influenced by the elastic scattering. We study the scattering effects introducing a shallow potential cavity in one of the arms of the ring. The scattering phase shift filters the resonances of the transferred momenta. We find that the AB os- 


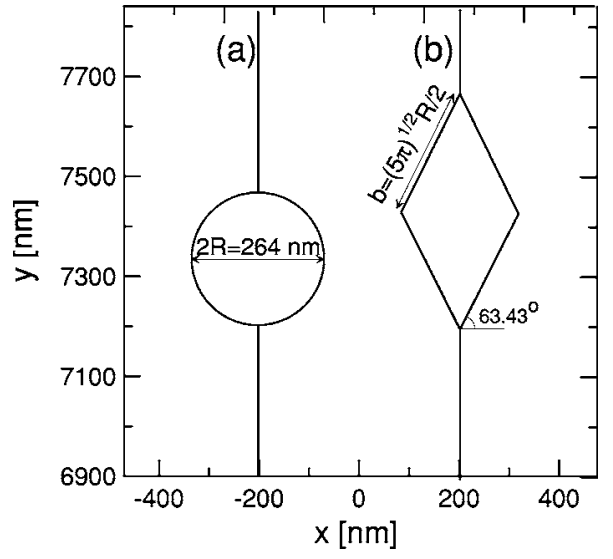

FIG. 1. Geometry and dimensions of the circular (a) and diamond (b) quantum rings studied in the present paper. In the calculations the positions of the centers of the Gaussians (2) are chosen along the drawn lines with a spacing of $20 \mathrm{~nm}$.

cillation of the packet transfer probability is shifted by $\Phi_{0} / 2$ only when the depth of the cavity is tuned such that it produces a $\pi$ phase shift for the maximum of the wave packet in the momentum space. For other depths we find the appearance of minima in the transition probability at integer multiples of $\Phi_{0} / 2$ that leads to a halving of the $\mathrm{AB}$ period, which was originally expected ${ }^{15}$ for strongly disordered quantum rings and recently observed in GaAs quantum rings. ${ }^{6}$

\section{THEORY}

We consider an electron confined in the $(x, y)$ plane with perpendicular magnetic field $(0,0, B)$. The Hamiltonian related to the kinetic energy has the form $H=\left(1 / 2 m^{*}\right)(-i \hbar \nabla$ $+e \mathbf{A})^{2}$ with the vector potential taken in the Landau gauge $\mathbf{A}=(-B y, 0,0)$ and $m^{*}$ stands for the electron effective mass (we take the GaAs value $m^{*}=0.067 m_{0}$ ). We expand the wave function in a basis of Gaussian functions ${ }^{16}$ centered around chosen points $\mathbf{R}_{n}=\left(X_{n}, Y_{n}\right)$

$$
\Psi(x, y, t)=\sum_{n} c_{n}(t) f_{n}(x, y),
$$

with

$$
\begin{aligned}
f_{n}(x, y)= & \exp \left[-\left(\mathbf{r}-\mathbf{R}_{n}\right)^{2} / 2 \lambda^{2}\right. \\
& \left.+i e B\left(x-X_{n}\right)\left(y+Y_{n}\right) / 2 \hbar\right] / \lambda \sqrt{\pi} .
\end{aligned}
$$

The shape of the considered structures is defined by the adopted position of centers $\left(\mathbf{R}_{n}\right)$ in functions (2). The centers are chosen along the lines drawn in Fig. 1 with spacings of $20 \mathrm{~nm}$. The parameter $\lambda$ in functions (2) is set to $19.8 \mathrm{~nm}$. That choice determines the width of the waveguides $(2 \lambda)$ and is equivalent to defining a harmonic oscillator confinement potential with the oscillator energy $\hbar \omega=2.9 \mathrm{meV}$ in the direction perpendicular to the waveguide. In the studied magnetic field range the increase of the electron localization in the wires with the magnetic field is negligible. ${ }^{17}$ The imaginary part of the exponent is related to the magnetic translation and ensures equivalence of all the points $\mathbf{R}_{n}$, i.e., the gauge invariance. Substituting expansion (1) into the timedependent Schrödinger equation we obtain a system of linear equations for the time derivative of the coefficients $c_{n}(t)$,

$$
\mathbf{S} \dot{\mathbf{c}}(t)=\mathbf{H c}(t) / i \hbar,
$$

where the elements of the overlap and Hamiltonian matrices are given by $\mathbf{S}_{k n}=\left\langle f_{k} \mid f_{n}\right\rangle$ and $\mathbf{H}_{k n}=\left\langle f_{k}|H| f_{n}\right\rangle$, respectively. However, the scheme based directly on Eq. (3) increases the amplitude of the wave function with each time step. A more stable and norm-conserving solution is provided by the Askar and Cakmak ${ }^{18}$ scheme producing a system of linear equations $\mathbf{S c}(t+d t)=\mathbf{S c}(t-d t)-2 i d t \mathbf{H c}(t) / \hbar$. Equation (3) is used only for evaluation of the first time step.

We consider circular and diamond rings enclosing an area of $132^{2} \pi \mathrm{nm}^{2}$ (see Fig. 1), for which a single flux quantum corresponds to $B=75.57 \mathrm{mT}$. For the incident wave packet we take one of the Gaussians (2), namely, the one localized at the wire at a position $y_{i}, 200 \mathrm{~nm}$ before the entrance of the ring multiplied by a plane wave, i.e.,

$$
\Psi(x, y, 0)=f_{i}(x, y) \exp (i q y) .
$$

This product is projected onto the basis (1) and the projection is used as the initial condition for the simulations. The corresponding probability density in wave vector space, calculated as a Fourier transform along the axis of the incoming lead $(x=0)$, is given by $P(k)=\sqrt{\pi} \sigma \exp \left[-(k-q)^{2} / \sigma^{2}\right]$ with $\sigma=0.0505 / \mathrm{nm}$. The flux of the $y$ component of the probability density current

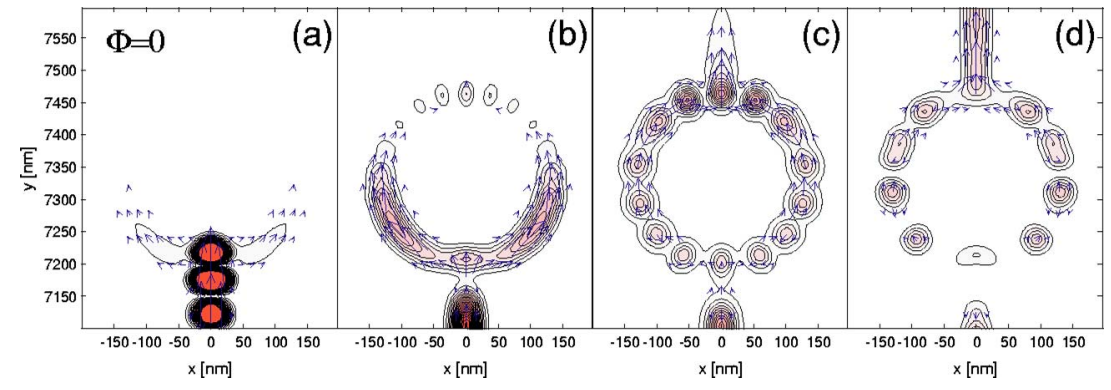

FIG. 2. (Color online) Charge (contours) and current (vectors) densities for a Gaussian wave packet with kinetic energy $\hbar^{2} q^{2} / 2 m$ $=1.42 \mathrm{meV}$ being transferred through a quantum ring of radius $132 \mathrm{~nm}$ for zero magnetic field. (a)-(d) correspond to $t=2.17$, 4.35, 6.35, and $8.8 \mathrm{ps}$. Scale for the charge and current density is the same in all the plots, with the exception of the current density vectors in (a) which were shortened by a factor of $1 / 2$ with respect to the other plots. 


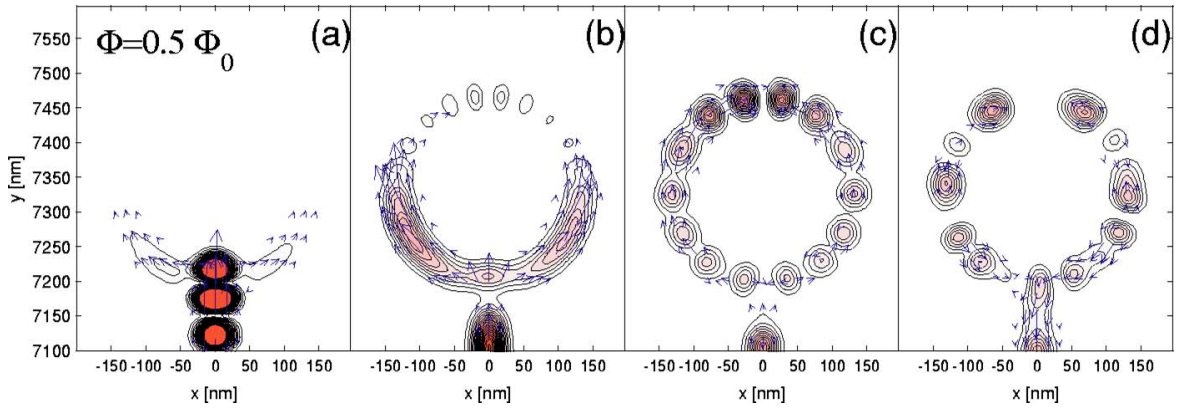

integrated over the two-dimensional space equals $\hbar q / \mathrm{m}^{*}$, which gives the same initial condition for all $B$. The central part of the packet in the wires moves in real space according to the Ehrenfest theorem as $d\langle\mathbf{r}\rangle / d t=\langle\mathbf{p}+e \mathbf{A}\rangle / m^{*}$, so in the applied gauge, for the leads oriented along the $y$ direction, the vector potential has no influence on the movement of the center of the wave packet. The Ehrenfest theorem for the change of the average momentum in time,

$$
\frac{d\langle\mathbf{p}+e \mathbf{A}\rangle}{d t}=-\frac{e}{m^{*}}\langle\mathbf{p}+e \mathbf{A}\rangle \times \mathbf{B},
$$

gives for the $y$ component the expression

$$
\frac{d\left\langle p_{y}\right\rangle}{d t}=\frac{d\langle-i \hbar \partial / \partial y\rangle}{d t}=-\frac{e B}{m^{*}}\left\langle i \hbar \frac{\partial}{\partial x}+e y B\right\rangle .
$$

The matrix elements of the operator $\left\langle f_{m}|i \hbar \partial / \partial x+e y B| f_{n}\right\rangle$ are zero for $X_{m}=X_{n}$ [see Eq. (2)], so in the leads (oriented parallel to the $y$ axis) the average value of momentum is conserved. In other words, the magnetic field cannot deflect the momentum of the electron packet moving in the leads defined as a sequence of Gaussian basis functions centered along the same axis. In that sense the leads in our model are effectively one-dimensional. Deflection is only possible at the junctions of the leads and the ring. Finally, we have verified using Fourier transform analysis that not only $\left\langle p_{y}\right\rangle$ and $\left\langle p_{y}^{2}\right\rangle$ but the entire momentum distribution remains unchanged in time when the wave packet travels through the leads. Summarizing, in our model the magnetic forces are not active in the leads, the momentum of the packet is conserved, although for $B \neq 0$ the momentum operator does not commute with the Hamiltonian.
The numerical results presented in this paper were obtained for an average value of the momentum $q=0.05 / \mathrm{nm}$, which corresponds to an average kinetic energy $\hbar^{2} q^{2} / 2 m^{*}$ $=1.42 \mathrm{meV}$. The average kinetic energy is equal to the Fermi energy $\left(E_{F}=\hbar \pi n / m^{*}\right)$ of the two dimensional electron gas at the carrier concentration $n=0.4 \times 10^{11} / \mathrm{cm}^{2}$. At the higher energy end of the packet, i.e., for $k=q+\sigma$, the kinetic energy equals $5.36 \mathrm{meV}$.

\section{RESULTS}

Figures 2-4 show snapshots of the time evolution of the wave packet in a circular quantum ring [see Fig. 1(a)] for 0, 0.5 , and 4.5 flux quanta. The contour plots show the charge densities and the arrows display the probability density vectors (5). Plots for $t=2.17 \mathrm{ps}$ [in parts (a) of Figs. 2-4] correspond to the moment just before the maximum of the charge density packet enters the ring. A larger part of the wave packet is scattered back into the injection lead. The plot for $\Phi=0$ [Fig. 2(a)] shows an equal spreading of the wave packet into both arms of the ring. At $t=4.4$ and 6.5 ps [Figs. 2(b) and 2(c)] we observe the formation of a maximum at the exit region of the ring where left and right circulating parts of the packet meet. For $\Phi=\Phi_{0} / 2$ (Fig. 3) the parts of the packet transferred through the left and right arms interfere destructively [Figs. 3(b)-3(d)], leading to a zero charge density at the upper exit of the ring. Consequently, (almost) no charge is transferred out of the ring at this exit. The injection asymmetry due to the Lorentz force directing the wave packet to the left arm, visible already in Fig. 3(a), increases with the magnetic field (see Fig. 4 for $4.5 \Phi_{0}$ ). We observe also that for $4.5 \Phi_{0}$ the wave packet reaches further into the arm as compared to the effect at lower magnetic fields. The Lorentz force helps the higher-momentum parts of the packet enter into the ring instead of being reflected. In comparison to the case of $0.5 \Phi_{0}$ (Fig. 3), we see that due to the injection

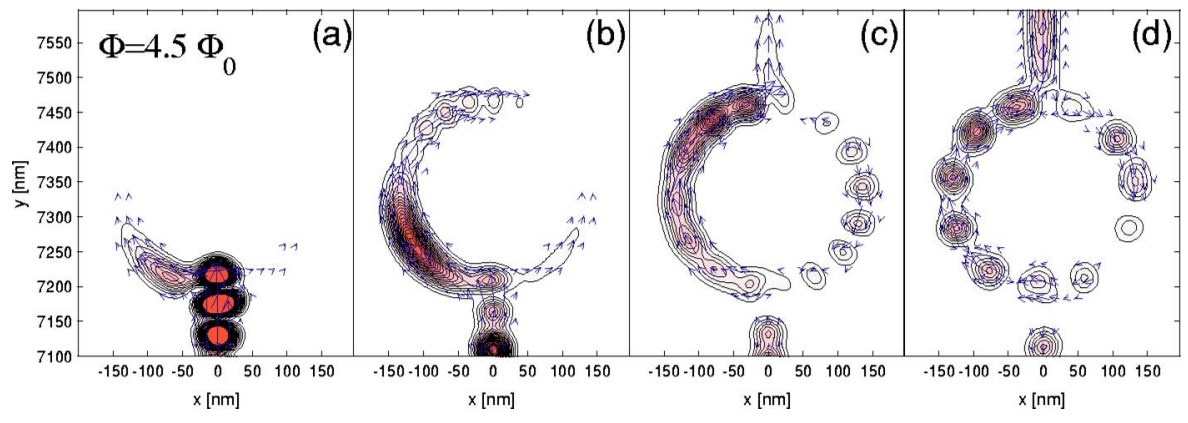

FIG. 4. (Color online) Same as Fig. 2 but for $B=0.34$ T, i.e., $\Phi$ $=4.5 \Phi_{0}$. 


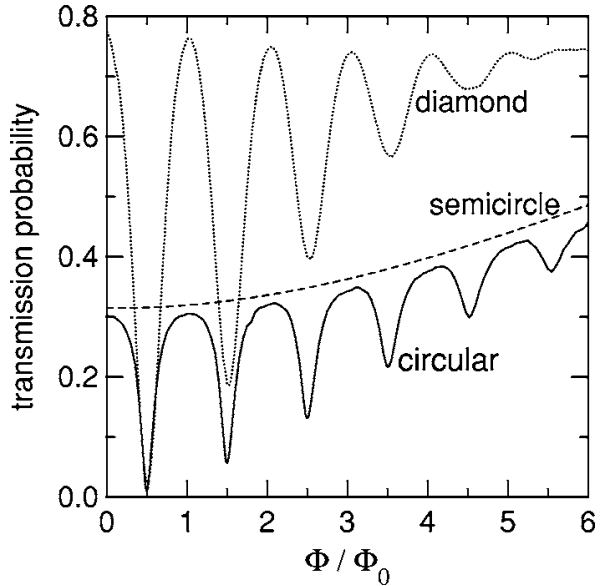

FIG. 5. The transmission probability of the wave packet through the circular (solid line), and diamond (dotted line) quantum rings as function of the flux passing through the ring in units of the flux quantum. The dashed line shows the transmission probability through a wire of semicircular shape obtained from the circular quantum ring after removal of one of its arms.

imbalance the destructive interference at the upper exit is not complete. The force also guides the packet, which travels throughout the left arm and exits the ring [Figs. 4(c) and $4(d)]$.

The transmission probability of the wave packet through the circular quantum ring is shown by the solid line in Fig. 5. This quantity was obtained by integrating the probability density leaving the ring through the upper lead. In contrast to the strictly one-dimensional model with the assumption of equal amplitudes of wave functions entering both arms of the ring as given by the Büttiker single-channel formula, ${ }^{9}$ we find (1) that the amplitude of the oscillations decreases with magnetic field, and (2) that for half-integer fluxes the value of the transmission probability is no longer zero. The decreasing amplitude is due to the growing imbalance in the amount of charge transferred through the left and right arms of the ring, which prevents the interference from being completely destructive. The values of the transmission probability maxima and minima are increasing functions of the magnetic field, which is a consequence of the guiding behavior of the Lorentz force that eases the entrance and exit of the wave packet. The envelope of the maxima is well approximated by the packet transfer probability through a semicircular wire that is obtained when the right arm of the circular ring is removed, plotted with the dashed line in Fig. 5. One could expect that the probability of transfer through the semicircular wire $[T(B)]$ will be larger for $B>0$, since then the Lorentz force tends to deflect the trajectories to the left. In fact, the transfer probabilities are independent of the magnetic field orientation $[T(B)=T(-B)]$. This is a signature of the microreversibility relation for a two-terminal device. ${ }^{19-21}$ The time dependence of the charge accumulated in the semicircular part of the wire, as well as the probabilities of finding the electron below and above the bend, are plotted in Fig. 6 for $\Phi=10 \Phi_{0}$. The probability density below the ring is at any time exactly the same for both the wires. The transmission probability tends for $t \rightarrow \infty$ to the same value for both

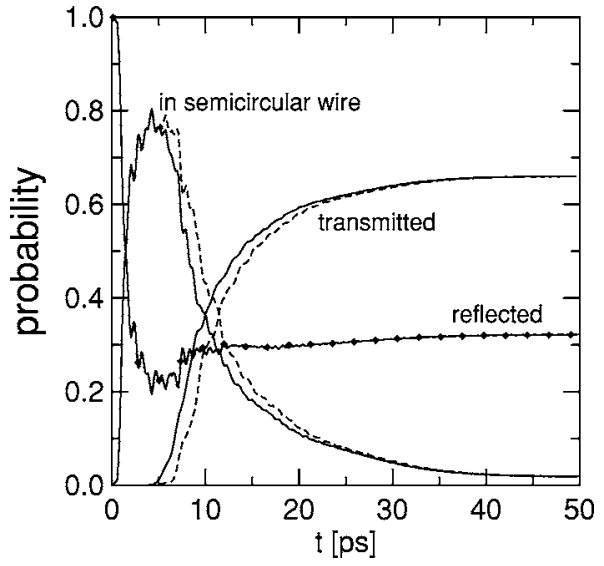

FIG. 6. Probability of finding the electron inside the semicircular wire above and below it as function of time for $\Phi=10 \Phi_{0}$. The solid (dashed) lines show the results for $B<0(B>0)$. The reflected probability density for the $B<0$ is marked by the dots.

wires, but the transport for $B<0$ is delayed with respect to the $B>0$ case. For $B>0$ the Lorentz force directly injects the electron into the bend and then ejects it to the outgoing lead. On the other hand, for $B<0$ both the magnetic-fieldassisted injection into and the ejection out of the wire bend are realized after the electron velocity changes its sign in reflection from the junctions at which the waveguide turns at the $90^{\circ}$ angle, hence the time delay.

More information on the nature of the transport is obtained from the momentum distribution of the transmitted wave packet, calculated numerically as the square of the absolute value of the space Fourier transform of the wave function transmitted through the ring calculated along the axis of the output lead $(x=0)$. The black solid line in Fig. 7 shows the momentum distribution of the transmitted packet for zero magnetic field (the momentum distribution of the incident packet is plotted by the dashed curve). The origin of the pronounced peaks can be understood from the transmission mechanism illustrated in Fig. 2. The momenta that have the highest probability to be transferred from the injection to the collection lead correspond to standing waves with maxima of

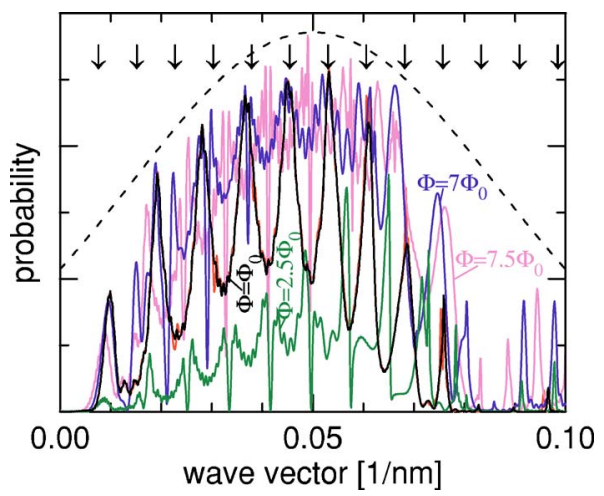

FIG. 7. (Color online) Probability density of the transferred wave packet through the circular ring in wave vector space. Lines corresponding to $0,1,2.5,7$, and 7.5 flux quanta passing through the ring are labeled. The dashed line shows the shape of the initial wave packet. The arrows show the wave vector values equal to $n / R$. 


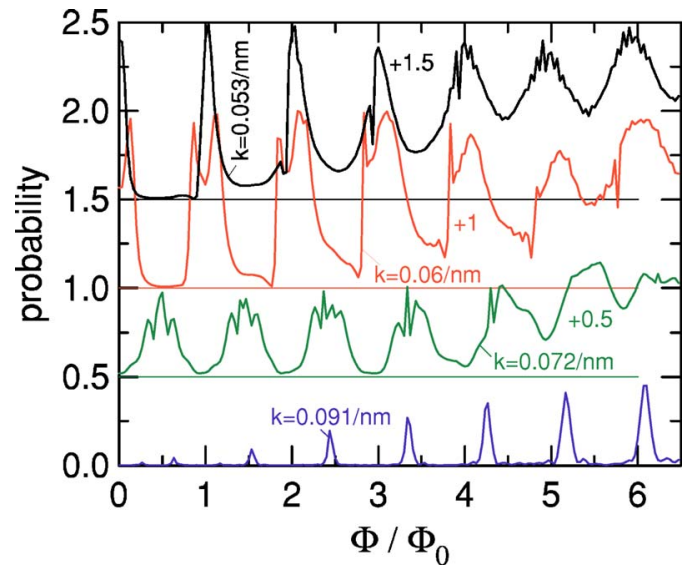

FIG. 8. (Color online) Transfer probability for fixed values of the incident wave vector as a function of the magnetic field flux for the circular ring. The plots for $k=0.072,0.06$, and 0.053 per nm were shifted by $0.5,1$, and 1.5 , respectively.

charge density at the entrance and the exit of the ring. This is realized when the phase shift $k \pi R$ along each of the arms is equal to an integer $(n)$ multiple of $\pi$ leading to the resonant condition $k=n / R$ (values marked by arrows in the top of the figure). Position of the momentum peaks for higher values of $k$ agrees well with these values. For lower momenta the spacing between the peaks increases, as if the resonant length for the slower parts of the packet was shorter. The transferred momentum spectrum for one flux quantum (see Fig. 7) is very similar to the one for zero magnetic field, and the position of the peaks is unaltered. For halves of the flux quanta at lower magnetic fields (see the plot for $2.5 \Phi_{0}$ ) the nonzero value for the transferred spectra is uniquely due to the injection imbalance. The spectrum possesses characteristic double-peak structure in between the maxima for integer flux quanta. For $\Phi=0$ we also notice a clear asymmetry in the transferred momentum with respect to its original distribution. The parts of the wave packet that travel faster have less time to enter into the arms of the ring before they get reflected back at the entrance to the ring into the incoming lead. The momentum distribution at $7 \Phi_{0}$ (blue curve) differs with respect to the $\Phi=0$ and $\Phi_{0}$ distributions in two points: (1) the gaps between the peaks are filled, and (2) a visibly larger probability of transfer of the fast parts of the packet. Consequently, the spectrum approaches more closely the initial momentum distribution. The Lorentz-force guided transport does not require formation of standing waves with maxima at the ring-leads junctions, which is the reason why the resonant relation no longer holds. At high field for fractional flux quanta (see the plot for $7.5 \Phi_{0}$ ) the minima in the transferred spectrum are shifted toward distinctly nonzero values.

Figure 8 shows the transfer probability as a function of the magnetic field for fixed values of the wave vector. For $k=0.053 / \mathrm{nm}$ and $0.06 / \mathrm{nm}$ the magnetic field leads to a decrease of the oscillation amplitude, as in the momentumaveraged packet transfer probability (see Fig. 5). The growth of the envelope of transfer probability maxima seen in Fig. 5 is due to the Lorentz-force guided transport for high incident momenta. For $k=0.072 / \mathrm{nm}$ the transfer probability grows

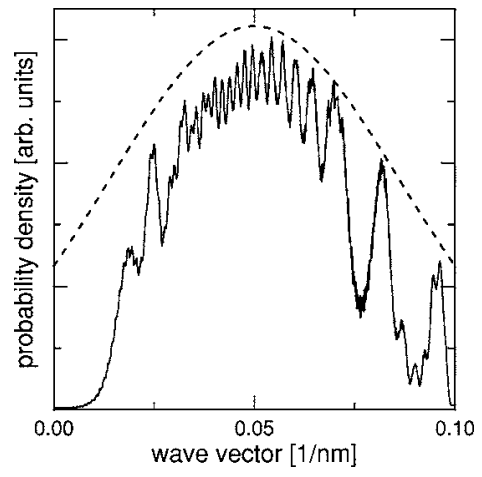

FIG. 9. Probability density of the packet transferred through the diamond ring in wave vector space for $B=0$. The dashed line shows the shape of the initial wave packet.

with decreasing $\mathrm{AB}$ oscillation amplitude. On the other hand, already for $k=0.091 / \mathrm{nm}$ the amplitude increases with $B$. This can be understood on the basis of the properties of the semicircular wire discussed above. The electron can be injected by the Lorentz force into the left arm of the ring directly from the incoming lead or to the right arm after its velocity changes sign at the reflection at the junction. For high momenta the Lorentz force first allows transport through both the arms of the ring. It should be expected that at higher $B$ the injection imbalance will appear and eventually destroy the $\mathrm{AB}$ oscillations. Note that the peaks of the transfer probability for $k=0.091 / \mathrm{nm}$ are spaced by only around $90 \%$ of the nominal $\mathrm{AB}$ period, which indicates that the effective radius of the ring is larger for fast electrons. This classical feature was already noticed in the enlarged spacings between the resonant peaks at the low- $k$ part of the spectrum for $B=0$ in Fig. 7 .

As compared to the circular ring, in the diamond geometry the incoming packet enters the arms of the ring more easily and leaves also more easily the ring to the upper lead. Consequently, at $B=0$ the transmission probability is more than twice larger than for the circular ring (see the dotted line in Fig. 5). No pronounced resonance pattern similar to the one obtained for the circular ring (compare Figs. 7 and 9) is observed. The transmitted momentum spectrum exhibits an asymmetric shift towards higher momenta with respect to the initial momentum distribution which is opposite to the circular ring case. The electrons with higher momenta are now more easily transferred through the diamond ring simply by the inertia and not by the Lorentz force. That explains why for the diamond ring the envelope of the maxima of the packet transfer probability (Fig. 5) does not exhibit the growth with $B$ as in the circular ring case. Since the angle at which the trajectory has to be deflected is smaller than for the discussed circular ring geometry, the minima in the oscillations increase much faster with the $B$ field as compared to the circular ring (see Fig. 5). For $\Phi>5 \Phi_{0}$ the AB oscillations in the diamond ring are no longer observed.

Next, we study the effect of elastic scattering on a shallow Gaussian potential cavity placed in the center of the left arm of the circular ring. To determine the scattering properties of the cavity we solved first the strictly one-dimensional problem of transmission through a Gaussian quantum well 


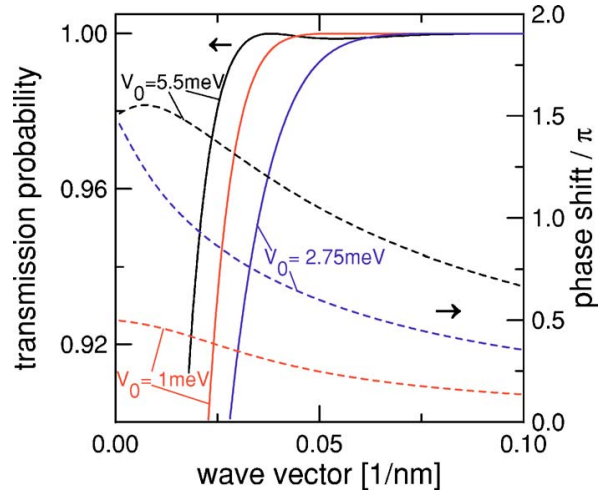

FIG. 10. (Color online) Transmission probability (solid lines) and the phase shift (dotted lines) as functions of the wave vector for a strictly one-dimensional Gaussian potential cavity of width $28 \mathrm{~nm}$ and different depths $V_{0}$.

$\left[-V_{0} \exp \left(-x^{2} / 2 \lambda^{2}\right)\right]$. Figure 10 presents the transmission probability and the phase shift as functions of the wave vector. The cavity is transparent for wave vectors larger than $0.025 / \mathrm{nm}$, only the phase is changed with respect to the $V_{0}$ $=0$ (no cavity) case. The phase shift for $k=0.05 / \mathrm{nm}$ (maximum of the probability density used in the time-dependent simulations) is close to $\pi / 4, \pi / 2$, and $\pi$ for $V_{0}=1,2.75$, and $5.5 \mathrm{meV}$, respectively.

In the time-dependent simulations for the ring structure we introduce a potential cavity described by the potential

$$
V(x, y)=-V_{0} \exp \left\{-\left[\left(x-X_{l}\right)^{2}+\left(y-Y_{l}\right)^{2}\right] / 2 \lambda^{2}\right\},
$$

where $\left(X_{l}, Y_{l}\right)$ is situated in the middle of the left arm of the ring. The transmission probability of the wave packet is plotted as a function of $V_{0}$ in Fig. 11(a) for different values of the flux. For $\Phi=0$ the transmission probability has a minimum when the phase shift for the maximum of the wave packet in momentum space is equal to $\pi$, which is achieved at $V_{0}$ $=5.5 \mathrm{meV}$ (cf. Fig. 10 for $q=0.05 / \mathrm{nm}$ ). Figure 11(b) shows the comparison of the momentum distribution of the transferred part of the packet for $V_{0}=0$ and $5.5 \mathrm{meV}$ at $\Phi=0$. The phase shift acquired in the dot and the destructive interference at the exit remove the central part of the wave packet in the momentum space. For $\Phi=\Phi_{0} / 2$ the cavity has the opposite effect on the transmission probability since it compensates for the $\pi$ shift produced by the $\mathrm{AB}$ effect. As a consequence the probability increases [see dashed lines in Fig. 11(a)] when $V_{0}$ is increased from 0 , and is maximal for $V_{0}$ $=5.5 \mathrm{meV}$ where the compensation of the $\mathrm{AB}$ phase shift is obtained. The central part of the transferred momentum spectrum at $\Phi=0.5 \Phi_{0}$ for $V_{0}=5.5 \mathrm{meV}$ [see Fig. 11(c)] is similar to $V_{0}=0$ in the absence of the magnetic field [compare black line in Fig. 11(b)]. Note, that the transmission probability plotted in Fig. 11(a) is not a smooth function of $V_{0}$. The changing slope of the curve is due to switching on and off the resonances in the transmitted momentum spectrum.

The magnetic field dependence of the packet transfer probability for $V_{0}=5.5 \mathrm{meV}$ is plotted in Fig. 12 by the lowest curve. The transfer probability possess maxima at the
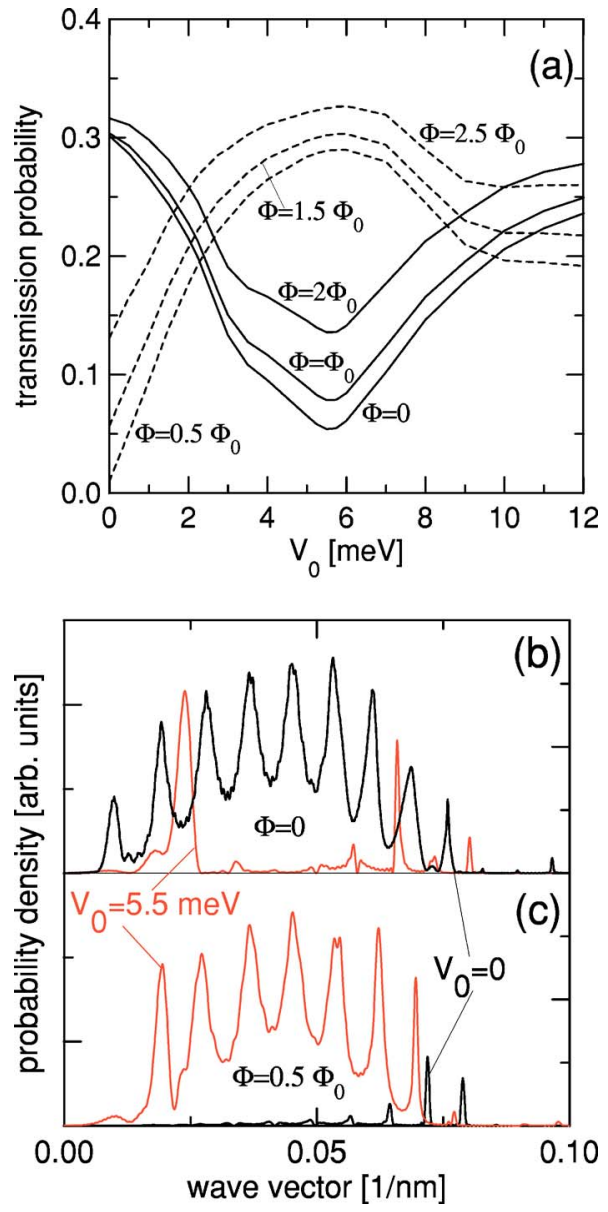

FIG. 11. (Color online) (a) Transmission probability of the wave packet through a circular ring with a Gaussian quantum well [Eq. (8)] in the left arm as a function of the depth of the well $V_{0}$. Values for fluxes equal to integer flux quanta are plotted with solid lines and for half flux quanta with dashed lines. (b) The transferred momentum distribution for $V_{0}=0$ and $5.5 \mathrm{meV}$ at $\Phi=0$. (c) Same as (b) but now for $\Phi=0.5 \Phi_{0}$.

magnetic fields corresponding to halves of the flux quanta, for which the Aharonov-Bohm effect compensates for the scattering $\pi$ shift. The transfer probability for $V_{0}=4 \mathrm{meV}$ is plotted by the second curve from below in Fig. 12. By analogy to the results for $V_{0}=0$ and $V_{0}=5.5 \mathrm{meV}$ one could expect a similar behavior with minima spaced by $\Phi_{0}$ but shifted on the flux scale. However, this would violate the even symmetry of the two-terminal device properties as a function of the external field. The transfer probability are subject to the phase-locking ${ }^{21}$ of the $\mathrm{AB}$ oscillations resulting in an extremum always present at $B=0$. For $V_{0}=4 \mathrm{meV}$ the transmission probability develops shallow minima at odd multiples of $\Phi_{0} / 2$. The probability amplitudes for the paths passing through the left and right arms do not meet exactly in phase at the upper exit from the wire, but on the other hand, the rotating left and right parts of the packet meet exactly in phase at the entrance. The depth of the probability minima for both the odd and even multiples of $\Phi_{0} / 2$ decrease with increasing flux. At $V_{0}=2.75 \mathrm{meV}$ the phase shift for the maximum of the wave packet $(q=0.05 / \mathrm{nm})$ is about $\pi / 2$ (see Fig. 10). The minima at the even and odd multiples of 


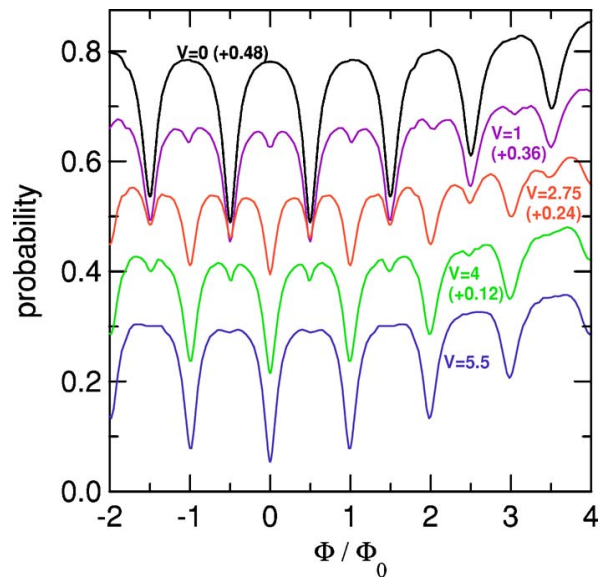

FIG. 12. (Color online) Probability of transmission of the wave packet through a circular ring with a Gaussian quantum well [Eq. (8)] in the left arm as a function of the magnetic field flux for well depths: $V_{0}=5.5,4,2.75,1$ and $0 \mathrm{meV}$. Lines for $V_{0}=4,2.75,1$, and $0 \mathrm{meV}$ have been shifted for clarity by $+0.12,+0.24,+0.36$, and +0.48 , respectively.

$\Phi_{0} / 2$ acquire similar depth. As a consequence the transmission probability exhibits oscillations with an effective quasiperiod of half of the flux quantum.

\section{DISCUSSION}

The measured conductance of semiconductor rings $3,4,6,7,22$ deviates from the strict periodicity predicted by the onedimensional models. ${ }^{8-10,19-21}$ We indicate that the Lorentz force can be responsible for these deviations. The injection imbalance leads to a decrease of the AB oscillations amplitude with the magnetic field as obtained in the magnetoconductance measurements ${ }^{6}$ performed on GaAs/AlGaAs quantum rings (see Fig. 1 of Ref. 6). The amplitude decreasing with magnetic field was also observed in GaAs/AlGaAs nanorings formed by an atomic force microscope tip ${ }^{4}$ [see the ring current plot in Fig. 2(c) of Ref. 4] and in the AB interferometer [see Fig. 3(a) of Ref. 22]. A suppression of the periodic magnetoresistance oscillations was also reported in Ref. 3. Lorentz force can also be responsible for an increase of the oscillations' amplitude (observed for instance in Ref. 7) since in some geometries it opens the transport window for fast electrons.

In order to be of significant importance the Lorentz force has to deflect the electron trajectories at the entrance and at the exit leads of the ring. The classical formula for the radius of an electron orbit in a magnetic field $R=m^{*} V / e B$ (for the average $q=0.05 / \mathrm{nm}$ taken in our calculations $R=32.9$ $\mathrm{nm} \mathrm{T} / B$ ) indicates that the effect will be smaller for fast electrons since the radius will not fit into the width of the junction. This feature was actually confirmed in the context of Figs. 7 and 8. In metals the Fermi energies are of order eV, which compares to meV in a two-dimensional electron gas in GaAs. Consequently, in $\mathrm{Au}$ rings ${ }^{2}$ in which both the electron effective mass $\left(\sim m_{0}\right)$ and the Fermi velocity $\left(1.4 \times 10^{6}\right.$ $\mathrm{m} / \mathrm{s}$ ) are about 15 times larger than the effective mass and the velocity $\left(\hbar q / m^{*}=0.086 \times 10^{6} \mathrm{~m} / \mathrm{s}\right.$ for $\left.q=0.05 / \mathrm{nm}\right)$ considered in the present paper, the $\mathrm{AB}$ oscillations pertain up to $8 \mathrm{~T}$ covering as much as $10^{4}$ flux quanta $\Phi_{0}$. Recently, AB oscillations in a semiconductor quantum ring ${ }^{5}$ pertaining to large fluxes were observed in a device with electrostatic tunnel barriers in both arms of the ring. In the experiment ${ }^{5}$ the barriers were tuned to have equal transmission, which can compensate for the Lorentz force effect described in the present paper.

The imbalance of the current through the arms of the ring as due to the magnetic field was previously ${ }^{14}$ found in a time-independent simulation. Our results contradict the proposed mechanism ${ }^{14}$ of the direction of the current injection changing from the left to the right arm of the ring periodically with the magnetic field. The injection imbalance is due to the Lorentz force; hence it is monotonic in $B$. At high field for single-channel transport the authors ${ }^{14}$ obtain the transmission probability as a bivalue function equal to zero for odd multiples of $\Phi_{0} / 2$ and 1 for other fluxes (see Fig. 13 of Ref. 14 for $\epsilon=20)$. According to our calculations there is no physical reason that would lead to a strict vanishing of the single-channel conductance at high magnetic field penetrating the arms of the two-dimensional quantum ring. The Hamiltonian eigenstates in the leads (oriented along $x$ axes) were used ${ }^{14}$ as boundary conditions for the incoming and outgoing waves. The eigenstates were calculated as $g_{k}(x, y)$ $=\exp (i k x) f_{k}(y)$, where $f_{k}$ is the eigenfunction of the onedimensional Hamiltonian $H=-\left(\hbar^{2} / 2 m^{*}\right) d^{2} / d y^{2}+\left(1 / 2 m^{*}\right)$ $\times\left(\hbar k-m^{*} \omega_{c} y\right)^{2}$ with zero boundary conditions on the wall, i.e., for $y= \pm d / 2$. The probability density current in the $x$ direction for eigenstate $g_{k}$ is given by $j_{x}(y)=|f(y)|^{2}(\hbar k / m$ $\left.-\omega_{c} y\right)$. At high magnetic field ${ }^{14}$ (when $\left.\omega_{c} \gg \hbar k / m^{*}\right)$ the current $j_{x}(y)$ has the opposite orientation at the top $(y>0)$ and bottom $(y<0)$ edges of both the injection and the extraction lead. ${ }^{23}$ Moreover, the conductance Landauer formula [Eq. (16) of Ref. 14] assumes that the current is proportional to the wave vector. This assumption is only correct at $B=0$.

\section{CONCLUSION AND SUMMARY}

We have solved the time-dependent Schrödinger equation for a Gaussian electron wave packet passing through a quantum ring in the presence of a homogeneous external magnetic field. In contrast to previous strictly one-dimensional theories our results indicate that the Aharonov-Bohm oscillations for semiconductor quantum rings disappear in the high-magnetic-field limit due to the Lorentz force action on the moving electron. In circular quantum rings the magnetic field changes the mechanism of transport, increasing the strength of the coupling of the ring to the leads with increasing magnetic field. The momentum resonances in the tunneling at low magnetic field resemble the formation of a quasibound temporary state localized in the ring. At high magnetic field the tunneling becomes guided by the Lorentz force. For rings, in which in the absence of the magnetic field the geometry blocks the transport of fast electrons, the Lor- 
entz force helps them to pass through. This can initially increase the amplitude of oscillations before the interference is eventually suppressed. The presence of an elastic scatterer in one of the ring arms leads to a $\pi$ shift of the oscillations of the wave packet transmission probability or to halving of the period of its Aharonov-Bohm oscillations.

\section{ACKNOWLEDGMENTS}

We are grateful to T. Ihn and K. Ensslin for helpful discussions. This research was supported by the Flemish Science Foundation (FWO-Vl) and the Belgian Science Policy. B.S. was supported by the EC Marie Curie IEF Project No. MEIF-CT-2004-500157.
${ }^{1}$ Y. Aharonov and D. Bohm, Phys. Rev. 115, 485 (1959).

${ }^{2}$ R. A. Webb, S. Washburn, C. P. Umbach, and R. B. Laibowitz, Phys. Rev. Lett. 54, 2696 (1985).

${ }^{3}$ G. Timp, A. M. Chang, J. E. Cunningham, T. Y. Chang, P. Mankiewich, R. Behringer, and R. E. Howard, Phys. Rev. Lett. 58, 2814 (1987).

${ }^{4}$ A. Fuhrer, S. Lüscher, T. Ihn, T. Heinzel, K. Ensslin, W. Wegscheider, and M. Bichler, Nature (London) 413, 822 (2001).

${ }^{5}$ W. G. van der Wiel, Yu. V. Nazarov, S. De Franceschi, T. Fujisawa, J. M. Elzerman, E. W. G. M. Huizeling, S. Tarucha, and L. P. Kouwenhoven, Phys. Rev. B 67, 033307 (2003).

${ }^{6}$ S. Pedersen, A. E. Hansen, A. Kristensen, C. B. Sorensen, and P. E. Lindelof, Phys. Rev. B 61, 5457 (2000).

${ }^{7}$ U. F. Keyser, C. Fühner, S. Borck, R. J. Haug, M. Bichler, G. Abstreiter, and W. Wegscheider, Phys. Rev. Lett. 90, 196601 (2003).

${ }^{8}$ Y. Gefen, Y. Imry, and M. Y. Azbel, Phys. Rev. Lett. 52, 129 (1984).

${ }^{9}$ M. Büttiker, Y. Imry, and M. Y. Azbel, Phys. Rev. A 30, 1982 (1984).

${ }^{10}$ M. Büttiker, Y. Imry, R. Landauer, and S. Pinhas, Phys. Rev. B 31, 6207 (1985).

${ }^{11}$ S. Viefers, P. Koskinen, P. Sing 'a Deo, and M. Manninen, Physica E (Amsterdam) 21, 1 (2004).
${ }^{12}$ S. Olariu and I. I. Popescu, Rev. Mod. Phys. 57, 339 (1985), and references therein.

${ }^{13}$ T. Usuki, M. Takatsu, R. A. Kiehl, and N. Yokoyama, Phys. Rev. B 50, 7615 (1994).

${ }^{14}$ K. N. Pichugin and A. F. Sadreev, Phys. Rev. B 56, 9662 (1997).

${ }^{15}$ B. L. Al'tshuler, A. G. Aronov, B. Z. Spivak, D. Yu. Sharvin, and Yu. V. Sharvin, JETP Lett. 35, 588 (1982).

${ }^{16}$ B. Szafran, F. M. Peeters, S. Bednarek, and J. Adamowski, Phys. Rev. B 69, 125344 (2004).

${ }^{17}$ Actually, the oscillator length (half of the waveguide width) is given by $l=\sqrt{\hbar / m^{*} \omega_{e}}$, where $\omega_{e}^{2}=\omega^{2}+\omega_{c}^{2} / 4$, where $\omega_{c}$ is the cyclotron frequency. For $B=0,0.5$, and $0.75 \mathrm{~T}, l=19.8,19.7$, and $19.6 \mathrm{~nm}$, respectively.

${ }^{18}$ A. Askar and A. C. Cakmak, J. Chem. Phys. 68, 2794 (1978).

${ }^{19}$ M. Büttiker, Phys. Rev. Lett. 57, 1761 (1986).

${ }^{20}$ M. Büttiker, Phys. Rev. B 38, 9375 (1988).

${ }^{21}$ A. L. Yeyati and M. Büttiker, Phys. Rev. B 52, R14360 (1995).

${ }^{22}$ A. Yacoby, M. Heiblum, D. Mahalu, and H. Shtrikman, Phys. Rev. Lett. 74, 4047 (1995).

${ }^{23}$ For the magnetic length shorter than the channel width the lead eigenstates can be identified with the lowest Landau level. For the lack of correspondence between the wave vector quantum number $k$ and the current in the lowest Landau level; see, e.g., S. Datta, Electronic Transport in Mesoscopic Systems (Cambridge University Press, Cambridge, England, 1995). 